\title{
ABORDAGEM ÀS DOENÇAS SEXUALMENTE TRANSMISSÍVEIS EM UNIDADES BÁSICAS DE SAÚDE DA FAMÍLIA*
}

Lígia Maria Cabedo Rodrigues', Claudia Santos Martiniano², Ana Elisa Pereira Chaves ${ }^{3}$, Elisângela Braga de Azevedo ${ }^{4}$, Severina Alice da Costa Uchoa $^{5}$

\begin{abstract}
RESUMO: Este estudo objetivou verificar como acontece a abordagem às Doenças Sexualmente Transmissíveis pelos profissionais de saúde; trata-se de uma pesquisa descritiva, de caráter qualitativo. Os dados coletados em 2008, por meio de entrevista com médicos/enfermeiros das Unidades Básicas de Saúde da Família do Município de Campina Grande, Estado da Paraíba, e tratados por meio de análise de conteúdo. A abordagem às doenças sexualmente transmissíveis varia nas diferentes unidades; está centrada no modelo biomédico ou de vigilância em saúde; e a atenção volta-se, prioritariamente, para as mulheres. Constata-se a necessidade de mudanças na lógica de abordagem às doenças transmitidas pelo sexo, incluindo o público masculino.

PALAVRAS-CHAVE: Doenças sexualmente transmissíveis; Saúde da família; Assistência à saúde.

\section{THE APPROACH TO SEXUALLY TRANSMITTED DISEASES IN BASIC FAMILY HEALTH UNITS}

\begin{abstract}
This study aimed to study how health professionals approach sexually-transmitted diseases. It is descriptive research, qualitative in character. Data was collected in 2008 via interviews with doctors and nurses in Basic Family Health Clinics in Campina Grande county in the state of Paraíba, and analyzed with content analysis. The approach to sexually-transmitted diseases varies from clinic to clinic; it is based around the biomedical model or health vigilance; and attention is focussed primarily on women. The article notes the need for change in the reasoning behind the approach to sexually-transmitted diseases, to include the male population.
\end{abstract}

KEYWORDS: Sexually-transmitted diseases; Family health; Health assistance.

\section{ABORDAJE DE LAS ENFERMEDADES SEXUALMENTE TRANSMISIBLES EN UNIDADES BÁSICAS DE SALUD DE LA FAMILIA}

RESUMEN: Este estudio tuvo el objetivo de verificar de que modo ocurre el abordaje a las Enfermedades Sexualmente Transmisibles por los profesionales de salud. Es una investigación descriptiva, de carácter cualitativo. Los datos fueron recogidos en 2008, por medio de entrevista con médicos/enfermeros de las Unidades Básicas de Salud de la Familia del município de Campina Grande, estado de Paraíba, y tratados por medio del análisis de contenido. El abordaje de las enfermedades sexualmente transmisibles oscila en las distintas unidades; está centrado en modelo biomédico o de vigilancia en salud; y la atención se vuelve, de modo prioritario, para las mujeres. Se constata la necesidad de cambios en la lógica de abordaje a las enfermedades transmitidas por el sexo, haciendo la inclusión del público masculino.

PALABRAS CLAVE: Enfermedades sexualmente transmisibles; Salud de la familia; Asistencia a la salud.

\footnotetext{
*Artigo extraído do Trabalho de Conclusão do Curso de Graduação em Enfermagem da Universidade Estadual da Paraíba-UFPB. ${ }^{1}$ Enfermeira. Residente em Saúde da Família e da Comunidade na UFPB.

${ }^{2}$ Enfermeira. Mestre em Saúde Coletiva. Professora do Departamento de Enfermagem da UFPB.

${ }^{3}$ Enfermeira. Mestre em Enfermagem em Saúde Pública. Professora da Universidade Federal de Campina Grande/PB.

${ }^{4}$ Enfermeira. Mestre em Enfermagem. Membro do Grupo de Estudos e Pesquisas em Saúde Mental Comunitária da UFPB. Professora do Departamento de Enfermagem da Faculdade Maurício de Nassau.

${ }^{5}$ Médica. Doutora em Saúde Coletiva. Professora do Departamento de Saúde Coletiva e do Programa de Pós-Graduação em Ciências da Saúde da Universidade Federal do Rio Grande do Norte-UFRN. Líder do Grupo de Pesquisa Estudos e Saúde Coletiva da UFRN.
}

\author{
Autor correspondente: \\ Lígia Maria Cabedo Rodrigues \\ Universidade Federal da Paraíba \\ R. Lindolfo Gonçalves Chaves, 44 - 58051-200 - João Pessoa-PB-Brasil \\ E-mail: ligiacabedo@yahoo.com.br
}

Recebido: $14 / 09 / 10$ Aprovado: 17/02/11 


\section{INTRODUÇÃO}

Iniciado em 1994, o Programa Saúde da Família (atual Estratégia Saúde da Família-ESF) se apresenta como a estratégia que o Ministério da Saúde adotou para reorientar o modelo assistencial do Sistema Único de Saúde (SUS), a partir da Atenção Básica. Tratase de um processo em construção que tem em vista a reversão da modalidade de assistência em saúde predominante no Brasil, que é superespecializada, fragmentada e centrada na cura ${ }^{(1)}$. Esse procesos deve ser constituído por princípios fundamentais, como a integralidade, equidade e participação social.

Mediante a adstrição da clientela, as equipes da ESF estabelecem vínculos com a população, possibilitando o compromisso e a corresponsabilidade dos profissionais com os usuários e a comunidade ${ }^{(2)}$. Com papel preponderante na Saúde Coletiva, a ESF deve estar preparada para identificar agravos à saúde da população adstrita, sobretudo aqueles que já se constituem problema de Saúde Pública, como é o caso das Doenças Sexualmente Transmissíveis (DST).

Estas doenças, em geral, são transmitidas pela pessoa infectada aos seus parceiros, em relações sexuais desprotegidas, principalmente quando ocorre a penetração. Também podem ser transmitidas da mãe infectada para o bebê, durante a gravidez ou durante o parto, por transfusão de sangue contaminado ou compartilhamento de seringas e agulhas contaminadas. As principais DST são Aids, sífilis, gonorreia, clamídia, tricomoníase, herpes, hepatite $\mathrm{B}$, cancro mole e condiloma ${ }^{(3)}$.

Na atual organização da rede de serviços do SUS, a Unidade Básica de Saúde da Família (UBSF) corresponde à porta de entrada para o atendimento aos portadores dessas doenças. Portanto, cabe-lhe informar a população quanto às ações de prevenção, assisti-la de forma contínua e, quando necessário, encaminhá-la aos serviços de referência. As limitações dos planos de saúde (público ou privados) e dos programas preventivos, a que se justapõem fatores tais como o desconhecimento das doenças em geral e dos seus fatores de risco, levam as pessoas a procurar os serviços de saúde somente quando se tornam sintomáticas. As DST, entretanto, são geralmente assintomáticas e, com frequência, silenciosas. O rastreamento de indivíduos em risco, porém sem sintomas, é parte importante de qualquer estratégia de controle dessas doenças ${ }^{(4)}$.

Entre as principais ações do Programa Nacional de DST e de aids para a melhoria da qualidade do aten- dimento, destaca-se a implementação de uma política de assistência que garanta o acesso da população a uma rede básica de saúde que disponha de profissional capacitado, e a centros de referência para HIV e DST, quando em situações de maior complexidade diagnóstica ${ }^{(5)}$.

Com relação às ações da Atenção Básica, o Ministério da Saúde ${ }^{(6)}$ orienta que se deve incluir: atividades educativas para promoção à saúde e à prevenção; aconselhamento para os testes diagnósticos e para adesão à terapia instituída e às recomendações da assistência; diagnóstico precoce das DST, infecção pelo HIV e hepatites. Tratamento adequado da maioria das DST; encaminhamento dos casos que não competem a esse nível de atenção, realizando acompanhamento conjunto; prevenção da sífilis congênita e da transmissão vertical do HIV e manejo adequado dos indivíduos em uso indevido de drogas.

Na Atenção Básica, além das atividades de educação em saúde voltadas para a prevenção de DST, o manejo dos portadores dessas doenças e parceiros é realizado utilizando-se a abordagem sindrômica, que se baseia na identificação de sinais e de sintomas verificados no momento da avaliação clínica, por meio do uso de fluxogramas de conduta. Para as DST sintomáticas mais comuns, este é o método mais rápido empregado para identificar um agravo, e por meio do qual os indivíduos poderão ser tratados no momento da consulta. No entanto, o sucesso da abordagem sindrômica exige monitoração e avaliação constantes dos protocolos, bem como supervisão e treinamento do pessoal envolvido ${ }^{(7-8)}$.

A anamnese do paciente, a identificação das diferentes vulnerabilidades e o exame físico devem ser os principais elementos diagnósticos das DST. Destacase, entretanto, que para obter informações fidedignas para a realização de anamnese consistente e precisa, é necessário construir uma relação de confiança entre o profissional de saúde e o indivíduo em atendimento ${ }^{(9)}$.

É importante ressaltar que, apesar dos avanços na Atenção Básica nos últimos quinze anos, muitas UBSF ainda têm restrita capacidade resolutiva e trabalham com agendamento de consultas, destinando pouco ou nenhum espaço para atender a demanda espontânea, não reconhecendo as DST sintomáticas como uma emergência.

Isso restringe a acessibilidade aos serviços, levando, principalmente, os homens portadores de DST a continuar procurando atendimento em prontosocorros, farmácias, curandeiros ou mesmo recorren- 
do à automedicação. As mulheres, frequentemente assintomáticas, não são rastreadas ou orientadas em relação ao seu atendimento ginecológico ${ }^{(9)}$.

Considerando a importância do papel da ESF, como campo fértil para a promoção da saúde, tornase imprescindível saber como se dá a atuação dos profissionais de saúde no que diz respeito a esse grave problema de saúde pública. Sob tal enfoque, este estudo objetivou verificar como se dá a abordagem às DST na perspectiva dos profissionais atuantes nas Unidades de Saúde da Família de Campina Grande/PB.

\section{METODOLOGIA}

Trata-se de uma pesquisa descritiva, de caráter qualitativo, realizada em 2008 nas UBSF do Município de Campina Grande, Estado da Paraíba.

O universo foi composto por profissionais de nível superior que atuam na assistência aos usuários com diagnóstico de DST, nas referidas UBSF selecionadas para o estudo. Foi selecionada uma amostra do tipo estratificada e utilizados como subgrupos da população os Distritos Sanitários de Campina Grande. Para definir os profissionais que participariam da pesquisa, foi feito um sorteio aleatório simples de uma UBSF em cada um dos seis Distritos Sanitários, compondo uma amostra de seis Unidades da ESF. Assim, foram realizadas entrevistas com três enfermeiros e três médicos, observados os critérios de inclusão: ser médico ou enfermeiro com atuação há mais de dois anos e aceitar participar do estudo voluntariamente.

A coleta dos dados foi realizada por meio de entrevista, seguindo-se um roteiro com perguntas semiestruturadas; essa opção se deu por considerar que a fala desvenda condições estruturais de sistemas de valores, normas e símbolos, além de transmitir as representações de grupos determinados, em condições históricas, socioeconômicas e culturais peculiares ${ }^{(10)}$.

Como procedimento de análise qualitativa, utilizou-se a categorização, o que significa agrupar elementos, ideias ou expressões em torno de um conceito capaz de abrangê-los ${ }^{(11)}$. Os dados foram submetidos à Análise de Conteúdo, um conjunto de técnicas de análise das comunicações que, através da descrição do conteúdo das mensagens e dos indicadores (quantitativos ou não), permitem a inferência de conhecimentos acerca do contexto de produção/ recepção das mensagens ${ }^{(11)}$. Os discursos foram submetidos à análise, tendo sido realizadas várias leituras individualizadas para que, posteriormente, por meio da análise de conteúdo, fossem definidas as categorias.

O desenvolvimento do estudo foi aprovado e seguiu as diretrizes da Resolução 196/96 do Conselho Nacional de Saúde sob registro n. 1.877.0.000.133-08.

\section{RESULTADOS}

\section{Estratégia de realização da abordagem às DST}

A estratégia utilizada para abordar as DST em UBSF exerce fundamental influência na prevenção e no controle dessas doenças. Nas Unidades investigadas, foram encontradas duas situações que distinguem claramente a forma de concretização dessa atividade. A primeira é centrada no modelo biomédico, em que o elemento de mediação da relação médico-paciente é a doença traduzida em seus sintomas, como se denota no discurso a seguir:

Esta abordagem, nós realizamos quando o paciente já chega com alguma sintomatologia porque, inclusive, é muito atendimento aqui. Então, não dá para abordar todas as pessoas. Nós nos guiamos pela história do paciente, pelos sintomas. (Ent. 5)

Alguns modelos de estratégias de realização da abordagem às DST citados pelos profissionais entrevistados apresentam uma abordagem mais ampla, centrada na promoção da saúde:

Quando elas vêm para pegar o preservativo ou a pilula, a gente aborda DST, conversa e, também, fazemos abordagem das DST nos exames de lâmina, quando vamos realizar o citológico, principalmente com as mulheres que não estão mais na idade reprodutiva; quando elas vêm para o citológico, abordamos DST. (Ent. 3)

A gente começa, às vezes, em sala de espera, onde eu tenho meu manual, meu catálogo das DST, eu mostro as que são por corrimento, ferimento, o condiloma e até a Aids. E não falo só no Papanicolaou, que éo preventivo. A consulta também. Não pode esquecer se está sentindo alguma coisa, queixa relacionada principalmente à genitália. (Ent. 2)

\section{Frequência de abordagem às DST}

A importância de investigar, neste estudo, a frequência com que é realizada a abordagem às DST nas 
UBSF reside no fato de identificar como essas ações se inserem no contexto das equipes. Torna-se clara uma distinção entre as Unidades em relação à abordagem, com algumas baseadas na demanda espontânea:

Se ela chega aqui se queixando de uma dor de cabeça ou de outro sintoma, nós não abordamos, mas se chega aqui uma mulher se queixando de uma leucorreia, se queixando de uma dor pélvica, de uma dispaurenia ou mesmo o sexo masculino se queixando de uma secreção peniana ou de uma ferida, de uma úlcera genital, então, nós abordamos, orientamos, tratamos e encaminhamos. (Ent. 5)

Outros depoimentos evidenciam uma abordagem mais ampliada, na qual a oferta programada é o que norteia a assistência às DST:

[...] De certa forma, praticamente todos os dias a gente faz esse tipo de abordagem; se for a mãe, uma mulher que venha pra consulta, ali durante a sala de espera, a gente falando da DST, do uso de camisinha, de qualquer maneira a gente tá entrando na abordagem das DST. (Ent. 2)

\section{Profissionais que realizam abordagem às DST}

Nesse grupo, os entrevistados apontam o médico e o enfermeiro como os profissionais que mais tratam da abordagem às DST, talvez por serem os que fazem a assistência direta a essas doenças, como corroboram os relatos a seguir:

Eu acredito que médico e enfermeiro abordam. Eu acredito que ela [a médica] também aborde, porque ela trata. Tem algumas situações que eu encontro que eu passo pra ela, para ela tratar e algumas que ela encontra que é de orientação que ela passa para mim. (Ent. 3)

O estudo evidenciou ainda que em alguns serviços há o envolvimento de outros profissionais:

Os agentes de saúde, a médica, a enfermeira, a auxiliar e a dentista participam da abordagem às DST. Os agentes participam de ações educativas. Eles estudam, nós marcamos no dia de planejamento. $O$ assunto do mês é divido para quem vai fazer sala de espera, ai escolhe o assunto, eles estudam e falam. (Ent. 4)

\section{Usuários envolvidos na abordagem às DST}

Este estudo também demonstrou que é imprescindível saber que usuários são envolvidos nas ações voltadas para as DST. A esse respeito, pode-se observar o relato:

Na saúde vem muita coisa de programa de cima para baixo. Vem o governo, estipula trabalhar com idosos, trabalhar com mulheres, o planejamento, a parte materno-infantil, e não tem nada para o homem em geral. A gente faz em nível de consultório a triagem, que é a seleção da idade para câncer de próstata, um rastreamento, mas assim, não tem um trabalho feito para os homens. (Ent. 4)

Além das usuárias mulheres, existem outros grupos que também são frequentemente abordados sobre essa temática, como os adolescentes:

Adolescente, principalmente hoje com a liberdade sexual, com tudo, a camisinha fica um pouco até esquecida, só querem saber a questão de pílula. (Ent. 2)

Sabe-se que os jovens estão iniciando suas atividades sexuais cada vez mais cedo, o que resulta em gravidez indesejada e DST, entre elas, a aids. Por isso, abordar adolescentes quanto às DST é cada vez mais necessário na atualidade. Há também relatos quanto à atuação independente de faixa etária:

Não tem uma idade certa. Depende se já tem uma vida sexual ativa. (Ent. 5)

Todos eles são abordados na primeira consulta. (Ent. 6)

Os relatos acima expressam que os usuários são atendidos em relação às DST, o que representa que essa é uma atividade rotineira e essencial na prevenção e no controle a destas doenças.

\section{Contribuição dos profissionais para minimizar as DST como problema de Saúde Pública}

Sobre esse aspecto, a pesquisa mostrou que os problemas relativos à Saúde Pública podem ser reduzidos por meio da educação em saúde e do tratamento das DST: 
A contribuição se dá principalmente através da questão da educação em saúde mesmo. A gente poder fazer com que o usuário entenda a necessidade do uso do preservativo. (Ent. 1)

A minha contribuição é educação em saúde. Eu acho que a educação em saúde é fundamental, não só nas DST, mas em todo contexto de saúde; a minha contribuição é a orientação, informação e a promoção em saúde. (Ent. 3)

Bem, a minha colaboração é ouvir o paciente, examiná-lo e passar o devido tratamento. Orientar, pedir os exames, fazer o devido aconselhamento, convocar os parceiros para que venham aqui também e encaminhar quando eu vejo que dentro das minhas limitações eu não tenho como resolver, encaminhar para os colegas especialistas. (Ent. 5)

As distinções das respostas estão associadas ao foco de trabalho realizado em cada equipe com relação às DST.

\section{DISCUSSÃO}

O estudo revelou que, em alguns casos, as DST são abordadas por meio do atendimento curativista, voltado para a doença já instalada. Esse tipo de prática centra-se na atenção médica, excluindo componentes coletivos e sociais fortemente presentes nas DST.

Outros relatos, para além do modelo biomédico, apontam para uma prática sanitária alternativa, denominada de Vigilância em Saúde. Nesse caso, as UBSF priorizam o desenvolvimento de ações de prevenção primária (promoção da saúde, prevenção de doenças e assistência à saúde) com a população, pois, dessa forma, os profissionais estariam democratizando o conhecimento acerca do problema das DST e as formas de prevenção que podem ser realizadas. Essa forma de abordagem se apresenta como resposta social aos problemas de saúde, norteada pelo paradigma da produção social da saúde ${ }^{(12)}$.

Os sujeitos da pesquisa expressam qual a real função da Equipe de Saúde da Família que, além de acompanhar os usuários por meio de consultas, executa ações preventivas através da promoção da saúde e da proteção específica, buscando os usuários antes mesmo de proferirem a queixa. Dessa forma, aproxima-se do paradigma da Vigilância em Saúde, que resulta da combinação de três tipos de ações: a promoção da saúde, a prevenção de enfermidades e acidentes e a atenção curativa ${ }^{(12)}$.

Quando analisada a frequência da abordagem às DST, evidencia-se que a assistência prestada ao homem e à mulher está relacionada à doença, ou seja, é necessário que os sinais e os sintomas já estejam instalados nos usuários para que haja a abordagem do profissional. É preciso que a equipe de saúde reflita sobre a importância de também trabalhar as ações de promoção da saúde e prevenção das DST, de forma contínua, nos diversos grupos formados na comunidade. Assim, os profissionais estarão contribuindo mais efetivamente para a redução da incidência dessas doenças.

Desse modo, constata-se que algumas equipes atentam para a necessidade de se realizar abordagens dirigidas às DST durante consultas com fins variados, um dos quais deve ser o do planejamento familiar. A prestação de serviços de planejamento familiar é um componente importante da assistência à saúde reprodutiva, pois tem o objetivo primordial de evitar a gravidez ou possibilitar o desejo de engravidar, e o objetivo secundário da consulta de planejamento familiar é a verificação e assistência a qualquer sintoma incomum relacionado à saúde reprodutiva. A entrevista típica para a coleta da história clínica questiona as usuárias sobre a ocorrência de sintomas anormais relacionados à menstruação, à atividade sexual e às doenças sexualmente transmissíveis ${ }^{(4)}$.

No que diz respeito aos profissionais que realizam a abordagem às DST, observa-se a integração da equipe multiprofissional de Saúde da Família, na qual há a participação dos profissionais nas atividades de educação em saúde, o que favorece a promoção da saúde, embora se perceba que a escolha do tema relativo ao desenvolvimento de tais atividades seja dos membros da equipe. Convém ressaltar que seria importante, também, ouvir as necessidades da população, para planejar as ações educativas que devem ser postas em prática. A Política Nacional da Atenção Básica refere, como característica do trabalho em UBSF, a realização de uma ação interdisciplinar e em equipe, integrando áreas técnicas e profissionais de diferentes formações ${ }^{(13)}$.

Entre os usuários, a mulher é mais comumente abordada sobre DST. É fato que, historicamente, o Ministério da Saúde centralizou mais programas e serviços na atenção à saúde da mulher. Além disso, os estudos epidemiológicos, ao longo dos anos, vêm demonstrando uma elevada morbimortalidade no 
grupo de homens, tanto pelas doenças crônicas degenerativas quanto pelas transmissíveis.

Portanto, é necessário rever como tem sido feita a assistência às DST, uma vez que, tratar patologias que envolvem o casal não permite uma abordagem que inclua apenas as mulheres no processo de adoecer e de ter saúde. O tratamento isolado de uma pessoa não resolve o problema da DST, já que deve incluir o parceiro. Os homens também precisam ser abordados, necessitam de esclarecimentos; precisam ser assistidos, acompanhados e tratados adequadamente nos serviços públicos de saúde.

Nesse sentido, as políticas de saúde existentes não têm contemplado questões relativas ao modelo de masculinidade existente, o que sugere um preconceito que se reflete não só nos homens, mas também na sociedade como um todo e nas formulações de políticas públicas. Isso também pode ser sentido em relação aos profissionais de saúde, às parceiras e à população em geral, que estranham quando um homem procura, de forma preventiva, um serviço de saúde ${ }^{(14)}$.

Somente depois de dezenove anos de implantação do SUS é lançada a Política Nacional de Atenção Integral à Saúde do Homem, reconhecendo os agravos do sexo masculino como um problema de saúde pública. Nessa política, os direitos sexuais e reprodutivos estão postos tanto na perspectiva de superar a restrição da responsabilidade sobre as práticas contraceptivas às mulheres, quanto no reconhecimento de que os homens são sujeitos de direitos.

Percebe-se que no grupo pesquisado a contribuição dos profissionais na redução da incidência das DST envolve dois importantes eixos: o da educação em saúde e do tratamento. É importante ressaltar que é por meio de atividades informativas e educativas que se promove maior sensibilização da comunidade para esta problemática, e o objetivo final da abordagem é o tratamento o mais precocemente possível das DST ${ }^{(6)}$.

Contudo, os profissionais devem utilizar tecnologias do cuidado para o acolhimento e a acessibilidade dos usuários como forma de garantir a participação dos mesmos como "elemento essencial no contexto das práticas de saúde"(15:613), respeitando os aspectos sociais, morais, religiosos, emocionais, entre outros.

Apesar do modelo inovador, visualizam-se muitas dificuldades para a realização da abordagem às DST por meio da ESF, o que evidencia a necessidade de se rever, inclusive, a formação dos profissionais para a atuação nesta Estratégia e quais as condições para que ocorra o desenvolvimento de atividades de promoção à saúde e de prevenção de doenças neste modelo de atuação.

\section{CONSIDERAÇÕES FINAIS}

A ESF representa um avanço na implantação do SUS. Com vistas a reorientar o modelo tradicional de assistência à saúde, tem sido uma importante referência na lógica dessa mudança, desempenhando papel de grande relevância no atendimento às ações de saúde da população adstrita a um serviço público.

Este estudo revelou que existem diferentes formas de abordagem em relação às DST; ressalta-se que, para reduzir a incidência desses agravos, são necessárias ações profissionais e organizacionais, em consonância com as diretrizes da ESF e voltadas às necessidades da população, incluindo a clientela masculina. Apesar da ESF se programar para o atendimento baseado na demanda organizada, faz-se necessária a abertura de espaços para atender também à demanda espontânea, visando o atendimento aos usuários que necessitam de urgência na assistência, como no caso do portador de DST.

A participação dos profissionais é fundamental quando a lógica da ESF é ampliar as condições de saúde e de vida da população usuária dos serviços, o que leva a uma alteração da prática do sistema de saúde. Assim, os trabalhadores das UBSF devem estar engajados nesse propósito, objetivando levar às pessoas essa mudança de atenção à saúde. No enfoque às DST deve haver educação constante para a comunidade, a fim de que haja compreensão de que prevenir é a principal forma de controlar esses agravos e minimizar sua incidência. Desse modo, as ações educativas devem ocorrer tanto para os usuários como para os trabalhadores, visando à reflexão crítica sobre o processo saúde-doença para reorientar a prática em saúde.

\section{REFERÊNCIAS}

1. Gaíva MAM, Silva AMN. O processo de implantação do programa de saúde da família no estado de Mato Grosso, Brasil: perspectiva de atores sociais da gestão estadual. Cogitare Enferm. 2008;13(4):57684.

2. Ministério da Saúde (BR). Departamento de Atenção Básica. Atenção Básica e Saúde da Família. Saúde da família. [acesso em 18 mar 2008]. Disponível: http://200.214.130.35/dab/atencaobasica.php 
3. Ministério da Saúde (BR). Coordenação Nacional de DST e Aids. Políticas e diretrizes de prevenção das DST/aids entre mulheres. 2003. [acesso em 22 jun 2008]. Disponível: http://bvsms.saude.gov.br/bvs/ publicacoes/cd04_19.pdf

4. Codes JS, Cohen DA, Melo NA, Teixeira GG, Leal AS, Silva TJ, et al. Detecção de doenças sexualmente transmissíveis em ambientes clínicos e não clínicos na cidade de Salvador, Bahia, Brasil. Cad Saúde Pública. 2006;22(2):325-34.

5. Ministério da Saúde (BR). Prevenção e Controle das DST/HIV na Comunidade / Rede Básica de Saúde. Relatório de execução. Atividades realizadas de setembro/96 à junho/98. Projeto BRA 001/92 - "AIDS I". 1998. [acesso em 22 jun 2008]. Disponível: http:// bvsms.saude.gov.br/bvs/publicacoes/50prevencao_ controle_rede.pdf

6. Ministério da Saúde (BR). HIV/Aids, hepatites e outras DST. Caderno de Atenção Básica nº 18. 2006. [acesso em 13 mai 2008]. Disponível: http://189.28.128.100/ dab/docs/publicacoes/cadernos_ab/abcad18.pdf

7. Nadal SR, Manzione CR. Identificação dos grupos de risco para as doenças sexualmente transmitidas. Rev Bras Coloproctol. 2003;23(2):128-9.

8. Carvalho NS. Bioética e doenças sexualmente transmissíveis DST. DST J Bras Doenças Sex Transm. 2003;15(2):57-61.

9. Ministério da Saúde (BR). Secretaria de Vigilância em Saúde. Programa Nacional de DST e Aids. Manual de Controle das Doenças Sexualmente Transmissíveis. Brasília: Ministério da Saúde; 2005.

10. Minayo MCS. O desafio do conhecimento - pesquisa qualitativa em saúde. $8^{\mathrm{a}}$ ed. São Paulo: Hucitec; 2004.

11. Bardin L. Análise de conteúdo. $7^{\mathrm{a}}$ ed. Lisboa: Edições 70; 1977.

12. Mendes EV. Uma agenda para a saúde. $3^{\text {a }}$ ed. São Paulo: Hucitec; 1996.

13. Ministério da Saúde (BR). Portaria n. 648/GM, de 28 de março de 2006. Dispõe sobre a Política Nacional de Atenção Básica. Diário Oficial da União [Internet] 28 mar 2006 [acesso em 21 out 2008]. Disponível: http://bvsms.saude.gov.br/bvs/ publicacoes/prtGM648_20060328.pdf

14. Braz M. A construção da subjetividade masculina e seu impacto sobre a saúde do homem: reflexão bioética sobre justiça distributiva. Ciênc Saúde Colet. [Internet] 2005;10(1) [acesso em 12 jun 2008]. Disponível: http:// www.scielosp.org/pdf/csc/v10n1/a10v10n1.pdf.

15. Pinho LB, Hermández AMB, Kantorki LP. O discurso sobre o acolhimento e a acessibilidade nos serviços comunitários de saúde mental. Cogitare Enferm. 2009; 14(4):612-9. 